\title{
Fatores associados à aceitação da vacina influenza entre trabalhadores de saúde: conhecimento, atitude e prática
}

\author{
Factors associated with the acceptance of the influenza vaccine \\ among health workers: knowledge, attitude and practice
}

Tiago Pereira de Souza (http://orcid.org/0000-0001-7444-2854) ${ }^{1}$

William Mendes Lobão (https://orcid.org/0000-0002-3474-2024) ${ }^{1}$

Carlos Antônio de Souza Teles Santos (https://orcid.org/0000-0003-0970-0479) ${ }^{1}$

Maria da Conceição Chagas de Almeida (http://orcid.org/0000-0002-4760-4157) ${ }^{1}$

Edson Duarte Moreira Júnior (https://orcid.org/0000-0002-7081-8348) ${ }^{1}$

${ }^{1}$ Instituto Gonçalo Muniz, Fiocruz. R. Waldemar Falcão 121, Candeal. 40296710 Salvador BA Brasil tiagopsouza2011@

hotmail.com

\begin{abstract}
Influenza vaccine is recommended for all health workers, but vaccination coverage remains unsatisfactory. A cross-sectional study that aimed to identify factors associated with influenza vaccination was carried out with health workers from a large Hospital Complex in Salvador, Bahia. A self-administered questionnaire was used based on the models "Knowledge, Attitudes and Practices" and "Health Belief Model". The dependent variable was the vaccination status against influenza in 2014, and the independent variables were sociodemographic factors, vaccine history, knowledge and attitudes about influenza/influenza vaccine. Logistic regression was used, odds ratio was calculated with $95 \%$ confidence intervals, adjusting for sex, age and occupation. The best multivariate model was chosen through backwards elimination and the Akaike Information Criterion. 755 workers participated. Influenza vaccine coverage was $61.5 \%$, being higher among nurses (69.0\%) and lower among physicians (49.1\%). The factors associated with being vaccinated against influenza were: knowing that even when healthy, one must vaccinate against influen$z a(O R=3.15 ; 95 \% C I: 1.74-5.71) ;$ knowing that the vaccine does not protect for many years (OR $=2.08 ; 95 \%$ CI:1.30-3.33); and not to be afraid of post-vaccine adverse effects (OR $=1.93 ; 95 \%$ CI: 1.26-2.95).

Key words Influenza vaccination, Determinants, Health workers, Knowledge, Attitudes
\end{abstract}

Resumo A vacina influenza é recomendada para todos trabalhadores de saúde, mas sua cobertura permanece insatisfatória. Estudo transversal que objetivou identificar fatores associados à vacinação contra influenza, realizado com trabalhadores de saúde de um grande Complexo Hospitalar de Salvador, Bahia. Utilizou-se questionário autoaplicável baseado nos modelos "Conhecimento, Atitudes e Práticas" e "Health Belief Model". A variável dependente foi a vacinação contra influenza em 2014, e as independentes representaram fatores sociodemográficos, histórico vacinal, conhecimentos e atitudes sobre influenza/vacina influenza. Usou-se regressão logística, calculouse odds ratio a intervalos de confiança de 95\%, ajustando para sexo, idade e profissão. Elegeu-se o melhor modelo multivariado através de eliminação retrógada e do Critério de Informação de Akaike. Participaram 755 trabalhadores. A cobertura da vacina influenza foi de $61,5 \%$, sendo maior entre enfermeiros $(69,0 \%)$ e menor entre médicos (49,1\%). Os fatores associados a vacinarse contra influenza foram: conhecer que mesmo estando saudável deve-se vacinar $(O R=3,15$; $\left.I C_{95 \%}: 1,74-5,71\right)$; saber que a vacina não protege por muitos anos $\left(O R=2,08 ; I C_{95 \%}: 1,30-3,33\right)$; e não temer efeitos adversos pós-vacinais $(\mathrm{OR}=$ 1,$\left.93 ; I C_{95 \%}: 1,26-2,95\right)$.

Palavras-chave Vacinação contra influenza, Determinantes, Trabalhadores de saúde, Conhecimento, Atitudes 


\section{Introdução}

No mundo, estima-se que anualmente ocorram de 3 a 5 milhões de casos graves de influenza, culminando em 250 a 500 mil óbitos, aumento das hospitalizações, e prejuízos sociais e econômicos ${ }^{1}$.

Por outro lado, a vacinação é uma ferramenta eficaz na prevenção da influenza e na redução dos casos graves e hospitalizações relacionados a essa doença ${ }^{2-4}$. Os trabalhadores de saúde constituem um dos grupos prioritários para os quais a vacina influenza é recomendada anualmente ${ }^{5}$; além da proteção individual, a vacinação destes indivíduos reduz, indiretamente, o adoecimento e a mortalidade dos pacientes assistidos por instituições de saúde $e^{6,7}$.

No entanto, estudos realizados em diferentes locais do mundo frequentemente evidenciam baixa cobertura da vacina influenza entre trabalhadores de saúde, o que pode repercutir em adoecimento, absenteísmo e transmissão de influenza para pacientes, comprometendo o adequado funcionamento dos serviços de saúde $e^{8-12}$.

Diversos fatores podem estar relacionados à vacinação contra influenza, e a identificação destes pode subsidiar a elaboração de políticas e estratégias que resultem na ampliação da cobertura vacinal. No Brasil, onde a vacina influenza é recomendada para todo trabalhador de saúde ${ }^{13}$, a produção científica voltada à identificação de fatores relacionados à vacinação deste grupo prioritário ainda é escassa ${ }^{14}$. Este estudo pretende estimar qual a cobertura da vacina influenza entre trabalhadores de saúde de um complexo hospitalar da cidade de Salvador, e identificar fatores associados à vacinação contra influenza.

\section{Métodos}

Trata-se de um estudo transversal, realizado com trabalhadores de saúde de um complexo hospitalar filantrópico de Salvador, onde em 2014, com apoio da Secretaria Municipal de Saúde, ocorreram campanhas de vacinação contra influenza em dois momentos: de 12 a 22 de maio, e de 15 a 17 de outubro (a vacina continuou sendo oferecida nas Unidades Básicas de Saúde até o mês de dezembro). A referida instituição, que está entre os maiores complexos de saúde do Brasil com atendimento exclusivo ao Sistema Único de Saúde (SUS), possui: três enfermarias de clínica médica, três unidades de longa permanência, duas de clínica cirúrgica, uma unidade de oncologia, um centro de tratamento intensivo (CTI) adulto, um hospital pediátrico (com três enfermarias e um CTI pediátrico), um centro geriátrico, um centro de atenção à pessoa com deficiência (CAPD), ambulatório, uma central de materiais esterilizados (CME), banco de sangue, laboratório de bioquímica e cozinha hospitalar. A instituição possui 1.005 leitos, realiza anualmente cerca de 16 mil internamentos, dois milhões de atendimentos ambulatoriais e 10 mil cirurgias.

Os participantes do estudo foram trabalhadores de saúde das seguintes categorias: enfermeiros, técnicos/auxiliares de enfermagem, médicos, fisioterapeutas, nutricionistas, técnicos de laboratório, trabalhadores da higienização, agentes hospitalares e copeiros. Os critérios de inclusão na pesquisa foram: 1) pertencer a alguma das categorias citadas anteriormente, e 2) estar em exercício profissional no período da coleta de dados. Os critérios de exclusão foram 1) ter começado a trabalhar na área de saúde a partir de janeiro de 2015 e não responder à questão sobre vacinação contra influenza no ano de 2014.

Os dados foram coletados entre os meses de janeiro e maio de 2015 durante reuniões/treinamentos que ocorrem todo mês com cada categoria profissional. $\mathrm{O}$ instrumento de coleta de dados foi um questionário estruturado autoaplicável, construído com base em outros utilizados anteriormente em estudos com temática e metodologia semelhantes, sendo realizadas adaptações ao contexto local e aos interesses do estudo. O instrumento foi submetido à análise de três juízes e pré-testado entre trabalhadores de saúde.

A variável dependente foi recebimento de vacinação contra influenza em 2014 (1-sim; 0-não) e as variáveis independentes, descritas a seguir, pertenceram a quatro domínios distintos: I) sociodemográfico: sexo, idade, profissão, setor de trabalho (onde trabalhou por maior tempo durante os 12 meses anteriores à pesquisa); II) conhecimentos sobre: benefícios da vacinação contra influenza (eficácia, duração da proteção); possíveis barreiras/malefícios da vacinação (segurança e efeitos adversos); susceptibilidade à influenza; gravidade, contagiosidade, formas de prevenção e etiologia da influenza; e grupos prioritários para a vacinação; III) atitude: existência ou não de medo de eventos adversos pósvacinais; IV) histórico vacinal: situação para as vacinas contra difteria e tétano $(\mathrm{dT})$ e hepatite $\mathrm{B}$.

A idade e o tempo de experiência profissional foram categorizados em faixas. A variável "Profissão" foi categorizada em: enfermeiros, médicos, técnicos/auxiliares de enfermagem, outros 
profissionais de saúde (fisioterapeutas, nutricionistas e técnicos de laboratório) e profissionais de apoio (higienização, agentes hospitalares e copeiros, que atuam em atividades que dão suporte/apoio aos serviços assistenciais). Os setores de trabalho foram agrupados em: clínica médica, clínica cirúrgica, clínica pediátrica, UTI (adulto e pediátrica), unidades de pacientes institucionalizados (centro geriátrico e CAPD), ambulatório, centro cirúrgico, setores onde há pouco/nenhum contato com pacientes (CME, laboratório, banco de sangue e cozinha hospitalar), mais de um setor, e sem informação.

A situação quanto à vacina dT foi categorizada em "adequada" (reforço há menos de 10 anos) e "inadequada" (não saber se recebeu ou nunca ter recebido o reforço, não recordar há quanto tempo recebeu, ou ter recebido há mais de 10 anos). A situação vacinal para hepatite $B$ foi classificada como "adequada" (já ter recebido três ou mais doses da vacina) ou "inadequada" (não saber se já recebeu ou nunca ter recebido alguma dose da vacina, não se recordar da quantidade de doses já recebidas, ter recebido menos de 3 doses).

Utilizou-se o modelo teórico de "Conhecimentos, Atitudes e Práticas" (CAP), que possibilita conhecer o que os indivíduos sabem a respeito de um assunto, como se sentem diante do mesmo, e de que maneira os conhecimentos e atitudes são traduzidos através das suas práticas $^{15}$. Também foi utilizado o modelo Health Belief Model (HBM), bastante usado na compreensão dos fatores que influenciam a aceitação ou recusa da vacina influenza ${ }^{11}$, segundo o qual a adesão a cuidados de saúde como a vacinação é influenciada pelas percepções da gravidade e da susceptibilidade a determinada doença, pela percepção dos possíveis benefícios e dos eventuais malefícios/barreiras que a ação de saúde pode ocasionar, e pelos fatores externos que podem dispor ou não à ação ${ }^{16,17}$.

Aplicou-se a análise descritiva para obtenção da frequência absoluta e percentuais das variáveis estudadas. A associação entre as variáveis independentes e a vacinação contra influenza foi verificada pelo teste qui-quadrado $\left(\chi^{2}\right)$ e modelo de regressão logística, mediante o qual obteve-se a odds ratio (OR), com intervalos de $95 \%$ de confiança (IC95\%). As variáveis que apresentaram valor de $\mathrm{p} \leq 0,20$ na análise bivariada foram selecionadas para compor o modelo multivariado inicial. Para seleção das variáveis do modelo multivariado final, utilizou-se o procedimento de eliminação retrógrada e o "Critério de Informação de Akaike" (AIC). As variáveis sexo, faixa etária e profissão foram utilizadas como variáveis de ajuste do modelo multivariado. Foram consideradas estatisticamente significantes as associações que apresentaram valor de $\mathrm{p} \leq 0,05$. Os dados foram digitados no programa EpiData versão 3.1, e as análises feitas através do Stata versão 13, licenciado no Instituto Gonçalo Moniz (IGM/Fiocruz-Bahia). O estudo foi aprovado pelo Comitê de Ética em Pesquisa do Hospital Santo Antônio, em 22/07/2014, e foram respeitados os princípios da ética em pesquisas envolvendo seres humanos, contidos na Declaração de Helsinque (e suas reformulações subsequentes) e também normatizados na Resolução CNS 466/12.

\section{Resultados}

Dos 820 indivíduos incluídos na pesquisa, 65 $(7,9 \%)$ foram excluídos, ficando a amostra composta por 755 trabalhadores de saúde. O estudo alcançou 57,7\% (113/196) dos enfermeiros que trabalham na instituição, 41,3\% (310/751) dos técnicos de enfermagem, 61,0\% (94/154) dos outros profissionais de saúde (fisioterapeutas, nutricionistas e técnicos de laboratório), e 51,5\% (122/237) dos trabalhadores do apoio (higienização, copeiros e agentes hospitalares). Não foi possível conhecer o percentual de médicos alcançado pelo estudo, pois a Instituição não informou o quantitativo desses profissionais.

$\mathrm{Na}$ Tabela 1, são apresentadas as características sociodemográficas e situação vacinal (Influenza, dT e hepatite B) dos trabalhadores de saúde.

Observa-se predominância de mulheres $(82,5 \%)$ e pessoas mais jovens - $44,6 \%$ dos indivíduos possuíam entre 19 e 29 anos, e apenas 5\% tinham 50 ou mais anos. A idade variou entre 19 e 86 anos, com média de 32,5 anos (desvio padrão $=8,6$ ).

Cerca de dois terços referiram cinco anos ou menos de experiência profissional na área da saúde, com 23,3\% trabalhando, no máximo, há 1 ano. $\mathrm{O}$ tempo médio de experiência foi de seis anos (desvio padrão $=7,0$ ), com valores variando entre 0,2 e 54 anos. Quanto à categoria profissional, observou-se maior predominância de técnicos de enfermagem $(41,4 \%)$, seguido de enfermeiros $(15,2 \%)$ e médicos $(14,7 \%)$.

A maior parte dos profissionais trabalhou nos 12 últimos meses anteriores a pesquisa, predominantemente na clínica médica $(38,2 \%)$ e no ambulatório $(10,0 \%)$. A maioria $(52,3 \%)$ referiu 
Tabela 1. Características sociodemográficas e história vacinal dos trabalhadores de saúde de um Complexo Hospitalar de Salvador, em 2014.

\begin{tabular}{|c|c|c|}
\hline Característica & $\begin{array}{c}\text { Total } \\
(\mathbf{n}=755)^{\star}\end{array}$ & $\%$ \\
\hline \multicolumn{3}{|l|}{ Sexo $(n=747)$} \\
\hline Feminino & 616 & 82,5 \\
\hline Masculino & 131 & 17,5 \\
\hline \multicolumn{3}{|l|}{ Faixa etária $(n=682)$} \\
\hline 19-24 anos & 72 & 10,6 \\
\hline 25-29 anos & 232 & 34,0 \\
\hline 30-34 anos & 180 & 26,4 \\
\hline 35-39 anos & 78 & 11,4 \\
\hline $40-44$ anos & 54 & 7,9 \\
\hline $45-49$ anos & 32 & 4,7 \\
\hline $50-54$ anos & 15 & 2,2 \\
\hline 55-59 anos & 12 & 1,8 \\
\hline$\geq 60$ anos & 7 & 1,0 \\
\hline \multicolumn{3}{|l|}{$\begin{array}{l}\text { Tempo de experiência } \\
(\mathrm{n}=730)\end{array}$} \\
\hline$<1$ ano & 86 & 11,8 \\
\hline 1 ano & 84 & 11,5 \\
\hline $2-5$ anos & 323 & 44,2 \\
\hline $6-10$ anos & 131 & 18,0 \\
\hline $11-15$ anos & 35 & 4,8 \\
\hline $16-20$ anos & 28 & 3,8 \\
\hline$\geq 21$ anos & 43 & 5,9 \\
\hline \multicolumn{3}{|l|}{ Função (n = 749) } \\
\hline $\begin{array}{l}\text { Técnico/auxiliar de } \\
\text { enfermagem }\end{array}$ & 310 & 41,4 \\
\hline Enfermeiro & 113 & 15,2 \\
\hline Médico & 110 & 14,7 \\
\hline Higienização & 85 & 11,3 \\
\hline Fisioterapeuta & 55 & 7,3 \\
\hline Copeiro & 31 & 4,1 \\
\hline Nutricionista & 22 & 2,9 \\
\hline Técnico de laboratório & 17 & 2,3 \\
\hline Agente hospitalar & 6 & 0,8 \\
\hline
\end{tabular}

recebimento do último reforço da vacina $\mathrm{dT}$ há menos de 10 anos (conforme recomendação), e $59,7 \%$ relataram situação adequada para a vacina hepatite B (três doses ou mais). A cobertura global da vacina influenza em 2014 foi de $61,5 \%$ (464/755). Os enfermeiros foram aqueles que mais se vacinaram contra essa doença $(69,0 \%)$, seguido dos trabalhadores do apoio $(65,6 \%)$; por outro lado, a categoria médica foi a que apresentou menor cobertura vacinal $(49,1 \%)$.

A Tabela 2 apresenta as características sociodemográficas e histórico vacinal dos indivíduos, segundo situação da vacina influenza em 2014.
Tabela 1. Características sociodemográficas e história vacinal dos trabalhadores de saúde de um Complexo Hospitalar de Salvador, em 2014.

\begin{tabular}{|c|c|c|}
\hline Característica & $\begin{array}{c}\text { Total } \\
(\mathbf{n}=755)^{\star}\end{array}$ & $\%$ \\
\hline \multicolumn{3}{|l|}{ Setor de trabalho $(\mathrm{n}=749)$} \\
\hline Clínica médica & 286 & 38,2 \\
\hline Ambulatório & 75 & 10,0 \\
\hline Clínica pediátrica & 57 & 7,6 \\
\hline Mais de um setor & 57 & 7,6 \\
\hline Clínica cirúrgica & 54 & 7,2 \\
\hline Sem informação & 50 & 6,7 \\
\hline Geriatria & 41 & 5,5 \\
\hline UTI adulto & 33 & 4,4 \\
\hline UTI pediátrica & 25 & 3,3 \\
\hline Centro Cirúrgico & 22 & 2,9 \\
\hline $\begin{array}{l}\text { Central de Material } \\
\text { Esterilizado }\end{array}$ & 17 & 2,3 \\
\hline Banco de sangue & 11 & 1,5 \\
\hline Laboratório & 10 & 1,3 \\
\hline $\begin{array}{l}\text { Centro de Acolhimento à } \\
\text { Pessoa com Deficiência }\end{array}$ & 6 & 0,8 \\
\hline Cozinha & 5 & 0,7 \\
\hline \multicolumn{3}{|l|}{$\begin{array}{l}\text { Vacina influenza em } 2014 \\
(\mathrm{n}=755)\end{array}$} \\
\hline $\operatorname{Sim}$ & 464 & 61,5 \\
\hline Não & 291 & 38,5 \\
\hline \multicolumn{3}{|l|}{$\begin{array}{l}\text { Vacina dT - reforço }(\mathrm{n}= \\
751)\end{array}$} \\
\hline $\begin{array}{l}\text { Não sabe se tomou/nunca } \\
\text { tomou }\end{array}$ & 98 & 13,1 \\
\hline $\begin{array}{l}\text { Já tomou, mas não lembra } \\
\text { há quanto tempo }\end{array}$ & 212 & 28,2 \\
\hline $\begin{array}{l}\text { Tomou há mais de } 10 \\
\text { anos }\end{array}$ & 48 & 6,4 \\
\hline $\begin{array}{l}\text { Tomou há menos de } 10 \\
\text { anos }\end{array}$ & 393 & 52,3 \\
\hline \multicolumn{3}{|l|}{$\begin{array}{l}\text { Vacina hepatite } B \text { - } n^{\circ} \text { de } \\
\text { doses }(n=730)\end{array}$} \\
\hline $\begin{array}{l}\text { Não sabe se tomou } \\
\text { alguma dose/nunca } \\
\text { tomou }\end{array}$ & 31 & 4,3 \\
\hline $\begin{array}{l}\text { Já tomou, mas não lembra } \\
\text { quantas doses }\end{array}$ & 168 & 23,0 \\
\hline Tomou menos de 3 doses & 95 & 13,0 \\
\hline Tomou 3 doses ou mais & 436 & 59,7 \\
\hline
\end{tabular}

*Varia conforme número de perdas (missing).

Profissionais do sexo feminino, com idade de 40 a 49 anos e 1 ano de experiência profissional foram os que mais se vacinaram contra influenza em 2014. Aqueles com situação adequada em relação às vacinas $\mathrm{dT}$ ou hepatite $\mathrm{B}$ vacinaram-se 
Tabela 2. Características sociodemográficas e história vacinal dos trabalhadores de saúde num Complexo Hospitalar de Salvador, segundo estado vacinal para influenza em 2014.

\begin{tabular}{|c|c|c|c|c|c|}
\hline \multirow{3}{*}{ Característica } & \multirow{3}{*}{$\begin{array}{c}\begin{array}{c}\text { Total } \\
(\mathbf{n}=755)^{\star}\end{array} \\
\mathbf{n}\end{array}$} & \multirow{2}{*}{\multicolumn{2}{|c|}{$\begin{array}{l}\text { Vacinados } \\
(\mathrm{n}=464)^{\star}\end{array}$}} & \multirow{3}{*}{$\begin{array}{l}\text { ORbruto } \\
\text { (IC 95\%) }\end{array}$} & \multirow{3}{*}{ p valor } \\
\hline & & & & & \\
\hline & & $\mathbf{n}$ & $\%$ & & \\
\hline \multicolumn{6}{|l|}{ Sexo $(n=747)$} \\
\hline Feminino & 616 & 384 & 62,3 & 1 & \\
\hline Masculino & 131 & 76 & 58,0 & $0,84(0,57-1,23)$ & 0,356 \\
\hline \multicolumn{6}{|l|}{ Faixa etária $(\mathrm{n}=682)$} \\
\hline 19-29 anos & 304 & 194 & 63,8 & 1 & \\
\hline 30-39 anos & 258 & 155 & 60,1 & $0,85(0,61-1,20)$ & 0,363 \\
\hline 40-49 anos & 86 & 59 & 68,6 & $1,24(0,74-2,07)$ & 0,412 \\
\hline$\geq 50$ anos & 34 & 18 & 52,9 & $0,64(0,31-1,30)$ & 0,216 \\
\hline \multicolumn{6}{|l|}{ Tempo de experiência $(n=730)$} \\
\hline$<1$ ano & 86 & 58 & 67,4 & 1 & \\
\hline 1 ano & 84 & 58 & 69,0 & $1,08(0,56-2,06)$ & 0,822 \\
\hline $2-5$ anos & 323 & 199 & 61,6 & $0,78(0,47-1,28)$ & 0,321 \\
\hline $6-10$ anos & 131 & 72 & 55,0 & $0,59(0,33-1,04)$ & 0,068 \\
\hline $11-15$ anos & 35 & 18 & 51,4 & $0,51(0,23-1,14)$ & 0,101 \\
\hline $16-20$ anos & 28 & 17 & 60,7 & $0,75(0,31-1,80)$ & 0,515 \\
\hline$\geq 21$ anos & 43 & 26 & 60,5 & $0,74(0,35-1,58)$ & 0,434 \\
\hline \multicolumn{6}{|l|}{ Função $(\mathrm{n}=749)$} \\
\hline Médico & 110 & 54 & 49,1 & 1 & \\
\hline Enfermeiro & 113 & 78 & 69,0 & $2,31(1,34-3,99)$ & 0,003 \\
\hline Técnico/auxiliar de enfermagem & 310 & 189 & 61,0 & $1,62(1,05-2,51)$ & 0,031 \\
\hline Outros profissionais de saúde $e^{\star \star}$ & 94 & 61 & 64,9 & $1,92(1,09-3,37)$ & 0,024 \\
\hline Apoio $^{\star * \star}$ & 122 & 80 & 65,6 & $1,98(1,17-3,35)$ & 0,012 \\
\hline \multicolumn{6}{|l|}{ Setor de trabalho $(\mathrm{n}=749)$} \\
\hline Ambulatório & 75 & 36 & 48,0 & 1 & \\
\hline Clínica médica & 286 & 173 & 60,5 & $1,66(1,00-2,77)$ & 0,052 \\
\hline Clínica cirúrgica & 54 & 29 & 53,7 & $1,26(0,62-2,53)$ & 0,523 \\
\hline Clínica pediátrica & 57 & 37 & 64,9 & $2,00(0,99-4,07)$ & 0,054 \\
\hline $\begin{array}{l}\text { Unidades de pacientes } \\
\text { institucionalizados }{ }^{\star * \star *}\end{array}$ & 47 & 29 & 61,7 & $1,75(0,83-3,67)$ & 0,141 \\
\hline UTI (adulto e pediátrica) & 58 & 36 & 62,1 & $1,77(0,88-3,56)$ & 0,108 \\
\hline Centro cirúrgico & 22 & 19 & 86,4 & $6,86(1,87-25,16)$ & 0,004 \\
\hline $\begin{array}{l}\text { Pouco/nenhum contato com } \\
\text { pacientes }\end{array}$ & 43 & 33 & 76,7 & $3,58(1,54-8,28)$ & 0,003 \\
\hline Mais de um setor & 57 & 32 & 56,1 & $1,39(0,69-2,77)$ & 0,355 \\
\hline Sem informação & 50 & 37 & 74,0 & $3,08(1,41-6,71)$ & 0,005 \\
\hline \multicolumn{6}{|l|}{ Reforço vacina dT $(\mathrm{n}=751)$} \\
\hline Inadequado & 358 & 209 & 58,4 & 1 & \\
\hline Adequado (há menos de 10 anos) & 393 & 251 & 63,9 & $1,26(0,94-1,69)$ & 0,123 \\
\hline \multicolumn{6}{|l|}{ Vacina hepatite B $(\mathrm{n}=730)$} \\
\hline Inadequado & 294 & 171 & 58,2 & 1 & \\
\hline Adequado (3 doses ou mais) & 436 & 280 & 64,2 & $1,29(0,95-1,75)$ & 0,099 \\
\hline
\end{tabular}

mais contra influenza que aqueles cuja situação vacinal estava inadequada. No entanto, a análise bivariada não evidenciou associação significante entre receber a vacina contra influenza, e o sexo, idade, tempo de experiência ou situação de outras vacinas recomendadas para trabalhadores 
de saúde $(\mathrm{p}>0,05)$. A profissão mostrou associação com o recebimento da vacina influenza ( $\mathrm{p}<0,05)$ : tendo como referência os médicos, a cobertura vacinal foi significativamente superior em todas as demais categorias, destacandose enfermeiros $(\mathrm{OR}=2,31$; IC95\%: 1,34 - 3,99) e trabalhadores do apoio $(\mathrm{OR}=1,98$; IC95\%: 1,17 - 3,35). Ter trabalhado nos últimos 12 meses predominantemente no centro cirúrgico ou em setores onde há pouco ou nenhum contato com pacientes foi um fator relacionado ao recebimento da vacina influenza de modo estatisticamente significante. Dos 504 indivíduos que não temiam os efeitos adversos pós vacinação contra influenza, 66,7\% receberam a vacina em 2014 e, entre os que temiam, a cobertura foi de apenas $52,2 \%$. Não temer os eventos adversos pós-vacinais mostrou-se associado à vacinação $(\mathrm{OR}=$ 1,84; IC95\%: 1,32 - 2,55).

Na Tabela 3, foram avaliados os julgamentos dos profissionais de saúde perante sentenças avaliativas dos conhecimentos acerca da influenza e da vacina influenza, segundo situação para essa vacina em 2014.
Entre aqueles que concordaram que a vacina influenza é segura, $64,2 \%$ receberam-na em 2014, enquanto que entre os que discordaram a cobertura vacinal foi de $44,4 \%$, havendo associação estatisticamente significante entre conhecer que esta vacina é segura e aceitar recebê-la (OR $=2,24$; IC95\%: 1,46-3,44). A cobertura vacinal foi significativamente superior entre os trabalhadores que sabiam que a vacina influenza é muito eficaz/funciona bem $(65,3 \%)$, em relação àqueles que discordaram dessa afirmação $(54,6 \%)$ - (OR $=1,56$; IC95\%: 1,14-2,14).

Entre os trabalhadores que corretamente discordaram que "quem tem boa saúde não precisa tomar a vacina da gripe", $63,7 \%$ vacinaram-se, confrontando com a cobertura vacinal de apenas $40,8 \%$ entre os que julgaram a informação incorretamente (OR = 2,54; IC95\%:1,54-4,18). Os profissionais que corretamente discordaram de que "a vacina da gripe protege por muitos anos" vacinaram-se mais contra influenza $(63,3 \%)$ que os demais $(53,2 \%)$ - essa diferença foi estatisticamente significante $(\mathrm{OR}=1,52$; IC95\%:1,03 $2,24)$.

Tabela 3. Conhecimentos dos trabalhadores de saúde de um Complexo Hospitalar de Salvador, segundo estado vacinal para influenza em 2014.

\begin{tabular}{|c|c|c|c|c|c|c|}
\hline \multirow[t]{2}{*}{ Declaração } & \multicolumn{2}{|c|}{$\begin{array}{c}\text { Total } \\
(\mathbf{n}=755)^{*}\end{array}$} & \multicolumn{2}{|c|}{$\begin{array}{l}\text { Vacinados } \\
(\mathrm{n}=464)^{*}\end{array}$} & \multirow[t]{2}{*}{$\begin{array}{l}\text { ORbruto } \\
\text { (IC 95\%) }\end{array}$} & \multirow[t]{2}{*}{$\begin{array}{c}\mathrm{p} \\
\text { valor }\end{array}$} \\
\hline & $\mathbf{n}$ & $\%$ & $\mathbf{n}$ & $\%$ & & \\
\hline \multicolumn{7}{|c|}{$\begin{array}{l}\text { A vacina da gripe é recomendada para todos os } \\
\text { trabalhadores de saúde }(V)(n=728)\end{array}$} \\
\hline Não concordo & 39 & 5,4 & 21 & 53,8 & 1 & \\
\hline Concordo (correto) & 689 & 94,6 & 430 & 62,4 & $1,42(0,74-2,72)$ & 0,286 \\
\hline \multicolumn{7}{|c|}{$\begin{array}{l}\text { Pessoas com doenças crônicas, como diabetes, asma } \\
\text { e insuficiência cardíaca, devem tomar a vacina da } \\
\text { gripe }(V)(n=730)\end{array}$} \\
\hline Não concordo & 72 & 9,9 & 43 & 59,7 & 1 & \\
\hline Concordo (correto) & 658 & 90,1 & 408 & 62,0 & $1,10(0,67-1,81)$ & 0,705 \\
\hline \multicolumn{7}{|c|}{ A vacina da gripe é segura $(V)(N=733)$} \\
\hline Não concordo & 99 & 13,5 & 44 & 44,4 & 1 & \\
\hline Concordo (correto) & 634 & 86,5 & 407 & 64,2 & $2,24(1,46-3,44)$ & 0,000 \\
\hline \multicolumn{7}{|c|}{$\begin{array}{l}\text { Vacinar trabalhadores de saúde pode prevenir a } \\
\text { transmissão de gripe para pacientes }(V)(n=736)\end{array}$} \\
\hline Não concordo & 120 & 16,3 & 73 & 60,8 & 1 & \\
\hline Concordo (correto) & 616 & 83,7 & 383 & 62,2 & $1,06(0,71-1,58)$ & 0,782 \\
\hline \multicolumn{7}{|c|}{$\begin{array}{l}\text { A vacina da gripe é muito eficaz / funciona bem } \\
(\mathrm{V})(\mathrm{n}=725)\end{array}$} \\
\hline Não Concordo & 238 & 32,8 & 130 & 54,6 & 1 & \\
\hline Concordo (correto) & 487 & 67,2 & 318 & 65,3 & $1,56(1,14-2,14)$ & 0,006 \\
\hline
\end{tabular}


Tabela 3. Conhecimentos dos trabalhadores de saúde de um Complexo Hospitalar de Salvador, segundo estado vacinal para influenza em 2014.

\begin{tabular}{|c|c|c|c|c|c|c|}
\hline \multirow[t]{2}{*}{ Declaração } & \multicolumn{2}{|c|}{$\begin{array}{c}\text { Total } \\
(\mathbf{n}=755)^{\star}\end{array}$} & \multicolumn{2}{|c|}{$\begin{array}{l}\text { Vacinados } \\
(\mathrm{n}=464)^{\star}\end{array}$} & \multirow{2}{*}{$\begin{array}{l}\text { ORbruto } \\
\text { (IC 95\%) }\end{array}$} & \multirow{2}{*}{$\begin{array}{c}\mathrm{p} \\
\text { valor }\end{array}$} \\
\hline & $\mathbf{n}$ & $\%$ & $\mathbf{n}$ & $\%$ & & \\
\hline \multicolumn{7}{|c|}{$\begin{array}{l}\text { Quem lava/higieniza as mãos e usa máscaras } \\
\text { corretamente não precisa tomar a vacina da gripe } \\
\text { (F) }(\mathrm{n}=736)\end{array}$} \\
\hline Concordo & 42 & 5,7 & 25 & 59,5 & 1 & \\
\hline Não concordo (correto) & 694 & 94,3 & 427 & 61,5 & $1,09(0,58-2,05)$ & 0,796 \\
\hline \multicolumn{7}{|c|}{$\begin{array}{l}\text { Quem trabalha na área de saúde está menos } \\
\text { exposto ao vírus da gripe }(\mathrm{F})(\mathrm{n}=733)\end{array}$} \\
\hline Concordo & 69 & 9,4 & 39 & 56,5 & 1 & \\
\hline Não concordo (correto) & 664 & 90,6 & 415 & 62,5 & $1,28(0,78-2,12)$ & 0,331 \\
\hline \multicolumn{7}{|c|}{$\begin{array}{l}\text { Quem tem boa saúde não precisa tomar a vacina da } \\
\text { gripe }(F)(n=740)\end{array}$} \\
\hline Concordo & 71 & 9,6 & 29 & 40,8 & 1 & \\
\hline Não concordo (correto) & 669 & 90,4 & 426 & 63,7 & $2,54(1,54-4,18)$ & 0,000 \\
\hline \multicolumn{7}{|c|}{$\begin{array}{l}\text { A gripe é uma doença pouco contagiosa } \\
\text { (transmissível })(\mathrm{F})(\mathrm{n}=734)\end{array}$} \\
\hline Concordo & 87 & 11,9 & 53 & 60,9 & 1 & \\
\hline Não concordo (correto) & 647 & 88,1 & 398 & 61,5 & $1,03(0,65-1,62)$ & 0,915 \\
\hline \multicolumn{7}{|c|}{$\begin{array}{l}\text { A vacina da gripe protege por muitos anos }(\mathrm{F})(\mathrm{n} \\
=739)\end{array}$} \\
\hline Concordo & 126 & 17,1 & 67 & 53,2 & 1 & \\
\hline Não concordo (correto) & 613 & 82,9 & 388 & 63,3 & $1,52(1,03-2,24)$ & 0,034 \\
\hline \multicolumn{7}{|c|}{$\begin{array}{l}\text { A gripe não mata pessoas jovens e saudáveis }(F)(n \\
=732)\end{array}$} \\
\hline Concordo & 186 & 25,4 & 121 & 65,1 & 1 & \\
\hline Não concordo (correto) & 546 & 74,6 & 331 & 60,6 & $0,83(0,59-1,17)$ & 0,283 \\
\hline \multicolumn{7}{|c|}{$\begin{array}{l}\text { A gripe não é uma doença grave, porque quase } \\
\text { nunca mata }(\mathrm{F})(\mathrm{n}=727)\end{array}$} \\
\hline Concordo & 193 & 26,5 & 119 & 61,7 & 1 & \\
\hline Não concordo (correto) & 534 & 73,5 & 330 & 61,8 & $1,01(0,72-1,41)$ & 0,973 \\
\hline \multicolumn{7}{|c|}{$\begin{array}{l}\text { Mulheres grávidas não devem tomar a vacina da } \\
\text { gripe }(\mathrm{F})(\mathrm{n}=719)\end{array}$} \\
\hline Concordo & 214 & 29,8 & 122 & 57,0 & 1 & \\
\hline Não concordo (correto) & 505 & 70,2 & 326 & 64,6 & $1,37(0,99-1,90)$ & 0,057 \\
\hline \multicolumn{7}{|c|}{$\begin{array}{l}\text { A vacina da gripe também protege contra resfriado } \\
\text { (F) }(\mathrm{n}=718)\end{array}$} \\
\hline Concordo & 226 & 31,5 & 136 & 60,2 & 1 & \\
\hline Não concordo (correto) & 492 & 68,5 & 306 & 62,2 & $1,09(0,79-1,50)$ & 0,606 \\
\hline \multicolumn{7}{|c|}{$\begin{array}{l}\text { Receber muito vento, chuva ou ficar em locais } \\
\text { muito frios podem causar gripe }(F)(n=733)\end{array}$} \\
\hline Concordo & 456 & 62,2 & 288 & 63,2 & 1 & \\
\hline Não concordo (correto) & 277 & 37,8 & 162 & 58,5 & $0,82(0,61-1,12)$ & 0,208 \\
\hline \multicolumn{7}{|c|}{$\begin{array}{l}\text { A vacina da gripe às vezes pode causar gripe }(F)(n \\
=734)\end{array}$} \\
\hline Concordo & 499 & 68,0 & 313 & 62,7 & 1 & \\
\hline Não concordo (correto) & 235 & 32,0 & 141 & 60,0 & $0,89(0,65-1,23)$ & 0,478 \\
\hline
\end{tabular}

${ }^{*}$ Varia de acordo com número de perdas (missing). 
$\mathrm{Na}$ análise multivariada (tabela 4), evidenciou-se que o conhecimento de que mesmo quem tem boa saúde precisa tomar a vacina influenza constitui o mais forte preditor da vacinação (OR = 3,15; IC95\%: 1,74 - 5,71).

Saber que a vacina influenza não protege por muitos anos também manteve-se como um fator associado à vacinação $(\mathrm{OR}=2,08$; IC $95 \% 1,30$ $3,33)$, assim como não ter medo dos efeitos adversos pós-vacinais $(\mathrm{OR}=1,93$; IC95\%: 1,26 $2,95)$. Houve associação em nível borderline entre conhecer a eficácia da vacina e a prática de vacinar-se (OR = 1,50; IC95\%: 0,99 - 2,28).

\section{Discussão}

A cobertura vacinal global da vacina influenza $(61,5 \%)$ foi superior à encontrada em alguns outros estudos nacionais semelhantes, que apontaram taxas de $43,2 \%$ e $51,3 \%{ }^{18,19}$, porém inferior à meta estabelecida pelo Ministério da Saúde do Brasil $(80,0 \%)$ e às taxas de cobertura reportadas por esse órgão para o Brasil, Bahia e Salvador em 2014 - 96,6\%, 90,2\% e 90,6\%, respectivamente ${ }^{20}$.
Estudos com trabalhadores de saúde em diferentes regiões do mundo apontam taxas de vacinação contra influenza variáveis, mas frequentemente são identificadas baixas coberturas ${ }^{21-26}$.

Os enfermeiros foram os trabalhadores que mais se vacinaram $(69,0 \%)$, e a associação entre pertencer a essa categoria profissional e ter maior cobertura vacinal (análise bivariada) também foi evidenciada por outros estudos ${ }^{8,12}$. Por sua vez, a cobertura mais baixa ocorreu entre os médicos $(49,1 \%)$, o que ratifica resultados apontados por alguns pesquisadores ${ }^{18}$, porém confronta outros que apontam relação entre ser médico e vacinarse mais ${ }^{27,28}$.

Tal qual apontado por Corace et al. ${ }^{29}$, conhecer que a vacina influenza é indicada mesmo para indivíduos previamente saudáveis mostrouse um preditor da vacinação. Segundo Stretcher e Rosenstock ${ }^{17}$, esse tipo de associação pode ter ocorrido porque compreender-se como indivíduo suscetível a determinado desfecho negativo estimula práticas adequadas de saúde, ao passo que desconsiderar esse risco reduz a chance de adoção de medidas de promoção, prevenção e proteção da saúde. Nesse contexto, alguns pes-

Tabela 4. Fatores associados e não associados à vacinação contra influenza entre trabalhadores de saúde de um Complexo Hospitalar de Salvador, em 2014 (modelo multivariado).

\begin{tabular}{|c|c|c|}
\hline \multirow{2}{*}{ Variável } & \multicolumn{2}{|c|}{ Análise multivariada } \\
\hline & OR $($ IC 95\%)* & p valor \\
\hline \multicolumn{3}{|l|}{ A vacina da gripe é segura $(\mathrm{V})$} \\
\hline Não concordo & 1 & \\
\hline Concordo (correto) & $1,61(0,89-2,90)$ & 0,113 \\
\hline \multicolumn{3}{|l|}{ A vacina da gripe é muito eficaz / funciona bem (V) } \\
\hline Não Concordo & 1 & \\
\hline Concordo (correto) & $1,50(0,99-2,28)$ & 0,059 \\
\hline \multicolumn{3}{|l|}{ Quem tem boa saúde não precisa tomar a vacina da gripe $(\mathrm{F})$} \\
\hline Concordo & 1 & \\
\hline Não concordo (correto) & $3,15(1,74-5,71)$ & 0,000 \\
\hline \multicolumn{3}{|l|}{ A vacina da gripe protege por muitos anos $(\mathrm{F})$} \\
\hline Concordo & 1 & \\
\hline Não concordo (correto) & $2,08(1,30-3,33)$ & 0,002 \\
\hline \multicolumn{3}{|l|}{ Medo de eventos adversos pós-vacinais } \\
\hline Sim & 1 & \\
\hline Não sei/não conheço & $0,74(0,33-1,68)$ & 0,475 \\
\hline Não & $1,93(1,26-2,95)$ & 0,002 \\
\hline \multicolumn{3}{|l|}{ Situação vacinal (combinação): dT (reforço) e hepatite B } \\
\hline $\begin{array}{l}\text { Não sabe se já tomou / nunca tomou as vacinas dT (reforço) e } \\
\text { hepatite B }\end{array}$ & 1 & \\
\hline $\begin{array}{l}\text { Já tomou as vacinas dT (reforço) e/ou hepatite B (ao menos uma } \\
\text { dose) }\end{array}$ & $2,46(0,85-7,09)$ & 0,096 \\
\hline
\end{tabular}


quisadores complementam que a justificativa de que possui um sistema imunológico fortalecido e eficaz tem desmotivado trabalhadores de saúde a se vacinar contra influenza ${ }^{28,30}$.

No presente estudo, observou-se associação significativa entre conhecer que a proteção da vacina influenza não dura muito tempo e a prática de recebê-la. Essa predisposição pode ocorrer porque, possivelmente, aqueles indivíduos que sabem que a proteção não é duradoura sentemse mais vulneráveis à influenza à medida que o tempo passa, e por isso tendem a vacinar-se mais que aqueles que desconhecem essa informação. No entanto, isso não é unanimidade entre os pesquisadores, e alguns estudos afirmam que conhecer o tempo de proteção da vacina influenza não interfere na sua aceitação $0^{31,32}$.

Embora a ocorrência de eventos adversos graves pós recebimento da vacina influenza seja rara $^{33-35}$, pesquisas apontam o temor dessas reações como importante fator que influencia na decisão de vacinar-se ou não ${ }^{36-38}$. Quanto a isso, no presente estudo observou-se que há associação significativa entre não ter medo das reações adversas pós-vacinais e aceitar se vacinar contra influenza, ratificando resultados encontrados por Rehbmann et al. ${ }^{39}$.

\section{Conclusão}

Este trabalho mostrou-se importante por abordar uma temática ainda pouco explorada pela literatura científica nacional, e por conseguir identificar fatores associados ao recebimento da vacina influenza, contribuindo com o entendimento de elementos envolvidos no processo de aceitação desta vacina por trabalhadores de saúde do país. Dessa maneira, pode contribuir com a elaboração de estratégias voltadas à ampliação da cobertura da vacina influenza entre os trabalhadores de saúde, grupo indispensável ao adequado funcionamento dos serviços de saúde.
Uma das limitações desse estudo foi seu desenvolvimento em uma única instituição de saúde, restringindo potencialmente a extrapolação dos resultados. No entanto, deve-se considerar que o estudo ocorreu em um complexo hospitalar de referência estadual e regional, onde um grande quantitativo de profissionais trabalha em diferentes áreas e especialidades, portanto, dotado de grande diversidade. Outra limitação foi a não conferência da carteira vacinal dos trabalhadores de saúde para confirmação da sua vacinação contra influenza em 2014, possibilitando a ocorrência de respostas "socialmente adequadas". No entanto, possivelmente isso tenha sido atenuado pela técnica de aplicação do questionário (autoaplicável, em vez de entrevista). Por fim, a utilização de uma amostra de conveniência dentro da instituição representa uma limitação, contudo, nas reuniões das diferentes categorias profissionais, o instrumento foi igualmente oferecido a todos os indivíduos presentes.

\section{Colaboradores}

TP Souza: concepção do projeto, desenho da metodologia, coleta dos dados, análise e interpretação dos dados, redação do artigo, aprovação da versão a ser submetida à Revista. CAST Santos, MCC Almeida, WM Lobão e ED Moreira Júnior: concepção do projeto, desenho da metodologia, análise e interpretação dos dados, revisão do artigo, aprovação final da versão a ser submetida à Revista. 


\section{Agradecimentos}

Ao Conselho Nacional de Desenvolvimento Científico e Tecnológico (CNPq) pela bolsa de mestrado concedida.

À Fundação de Amparo à Pesquisa do Estado da Bahia (Fapesb) pelo auxílio financeiro para digitação/confecção do banco de dados.

À Associação Obras Sociais Irmã Dulce, por ter permitido realizar a pesquisa em suas dependências.

\section{Referências}

1. World Health Organization (WHO). Influenza (Seasonal). Genebra: WHO; 2014. [Internet]. [acessado 03 Jan 2014]. Disponível em: http://www.who.int/mediacentre/factsheets/fs211/en/

2. Bridges CB, Thompson WW, Meltzer MI, Reeve GR, Talamonti WJ, Cox NJ, Lilac HA, Hall H, Klimov A, Fukumuda K. Effectiveness and cost-benefit of influenza vaccination of healthy working adults: A randomized controlled trial. JAMA 2000; 284(13):16551663.

3. Hayward AC, Harling R, Wetten S, Johnson AM, Munro S, Smedley J, Murad S, Watson JM. Effectiveness of an influenza vaccine programme for care home staff to prevent death, morbidity, and health service use among residents: cluster randomised controlled trial. BMJ 2006; 333:1241.

4. Cheng AC, Kotsimbos T, Kelly PM. Influenza vaccine effectiveness against hospitalisation with influenza in adults in Australia in 2014. Vaccine 2015; 33(51):73527356.

5. Center for Disease Control and Prevention (CDC). Influenza Vaccination Information for Health Care Workers. Atlanta: CDC; 2015. [Internet]. [acessado 07 Mar 2016]. Disponível em: http://www.cdc.gov/flu/ healthcareworkers.htm

6. Carman WF, Elder AG, Wallace LA, McAulay K, Walker A, Murray GD, Stott DJ. Effects of influenza vaccination of health-care workers on mortality of elderly people in long-term care: a randomised controlled trial. Lancet 2000; 355(9198):93-97.

7. Amodio E, Restivo V, Firenze A, Mammina C, Tramuto F, Vitale F. Can influenza vaccination coverage among healthcare workers influence the risk of nosocomial influenza-like illness in hospitalized patients? J. Hosp. Infect. 2014; 86(3):182-187.

8. Maltezou HC, Maragos A, Katerelos P, Paisi A, Karageorgou K, Papadimitriou T, Pierroutsakos IN. Influenza vaccination acceptance among healthcare workers: A nationwide survey. Vaccine 2008; 26(11):1408-1410.

9. Rehmani R, Memon JI. Knowledge, attitudes and beliefs regarding influenza vaccination among healthcare workers in a Saudi hospital. Vaccine 2010; 28(26):4283-4287.

10. Brien S, Kwong JC, Buckeridge DL. The determinants of 2009 pandemic A/H1N1 influenza vaccination: A systematic review. Vaccine 2012; 30(7):1255-1264.

11. Prematunge C, Corace K, McCarthy A, Nair RC, Pugsley R, Garber G. Factors influencing pandemic influenza vaccination of healthcare workers - A systematic review. Vaccine 2012; 30(32):4733-4743.

12. Alshammari TM, AlFehaid LS, AlFraih JK, Aljadhey HS. Health care professionals awareness of, knowledge about and attitude to influenza vaccination. $\mathrm{Vac}$ cine 2014; 32(45):5957-5961. 
13. Brasil. Ministério da saúde (MS). Informe Técnico: Campanha Nacional de Vacinação Contra a Influenza. Brasília: MS; 2014. [Internet]. [acessado 25 Out 2015]. Disponível em: http://www.sbim.org.br/wpcontent/uploads/2014/04/Informe_Campanha_Influenza-_-25_03_2014.pdf

14. Luna EJA, Gattás VL, Campos SRSLC. Efetividade da estratégia brasileira de vacinação contra influenza: uma revisão sistemática. Epidemiol Serv Saúde 2014; 23(3):559-575.

15. Santos SL, Cabral ACSP, Augusto LGS. Conhecimento, atitude e prática sobre dengue, seu vetor e ações de controle em uma comunidade urbana do Nordeste. Cien Saude Colet 2009; 16(Supl.1):1319-1330.

16. Janz NK, Becker MH. The health belief model: a decade later. Health Educ. Q 1984; 11(1):1-47.

17. Stretcher V, Rosenstock IM. The Health Belief Model. In: Glanz K, Lewis FM, Rimer BK, editors. Health Behavior and Health Education: Theory, Research and Practice. San Francisco: Jossey-Bass; 1997. p. 31-36.

18. Cavalcante RS, Jorge AMZ, Fortaleza CMCB. Predictors of adherence to influenza vaccination for healthcare workers from a teaching hospital: a study in the prepandemic era. Rev Soc Bras Med Trop 2010; 43(6):611-614.

19. Dinelli MIS, Moreira TNF, Paulino ERC, Rocha MCP, Graciani FB, Moraes-Pinto MI. Immune status and risk perception of acquisition of vaccine preventable diseases among health care workers. Am J Infect Control 2009; 37(10):858-860.

20. Brasil. Ministério da Saúde (MS). Campanha nacional de vacinação contra a gripe 2014 - coberturas vacinais. Brasília: MS; 2015. [Internet]. [acessado 2015 Out 10]. Disponível em: http://pni.datasus.gov.br/consulta_Influenza_14_selecao.asp?naofechar $=\mathrm{N} \& \mathrm{en}$ viar $=$ ok\&grupo $=$ todos \& faixa $=$ todos\&sel $=$ cobertu ras\&sel_parcial $=10 \&$ sel_parcial_csv $=10$

21. Nowalk MP, Lin CJ, Zimmerman RK, Fox DE, Raymund M, Tanis MD, Harper JD, Willis BC. Self-reported influenza vaccination rates among health care workers in a large health system. Am J. Infect Control 2008; 36(8):574-581.

22. Blank PR, Schwenkglenks M, Szucs TD. Vaccination coverage rates in eleven European countries during two consecutive influenza seasons. I Infect 2009; 58(6):446-458

23. Abu-Gharbieh E, Fahmy S, Rasool BA, Khan S. Influenza vaccination: healthcare workers attitude in three Middle East countries. Int J Med Sci 2010; 7(5):319325.

24. Aguilar-Díaz FC, Jiménez-Corona, Ponce-de-LeónRosales S. Influenza vaccine and healthcare workers. Arch Med Res 2011; 42(8):652-657.
25. Chen SC, Hawkins G, Aspinall E, Patel N. Factors influencing uptake of influenza A (H1N1) vaccine amongst healthcare workers in a regional pediatric centre: lessons for improving vaccination rates. Vaccine 2012; 30(2):493-497.

26. Torner N, Godoy P, Soldevila N, Toledo D, Rius C, Domínguez A. Estudio actitudes sobre vacunación antigripal en profesionales sanitarios de atención primaria de Cataluña. Atención primaria 2015; 48(3):192-199.

27. Barrière J, Vanjak D, Kriegel I, Otto J, Peyrade F, Estève M, Chamorey E. Acceptance of the 2009 A(H1N1) influenza vaccine among hospital workers in two French cancer centers. Vaccine 2010; 28(43)7030-7034.

28. Bellia C, Setbon M, Zylberman P, Flahault A. Healthcare worker compliance with seasonal and pandemic influenza vaccination. Influenza and other Respiratory Viruses 2013; 7(Supl. 2):97-104.

29. Corace K, Prematunge C, McCarthy A, Nair RC, Roth V, Hayes T, Suh KN, Balfour L, Garber G. Predicting influenza vaccination uptake among health care workers: What are the key motivators? Am J Infect Control 2013; 41(8):679-684.

30. Tanguy M, Boyeau C, Pean S, Marijon E, Delhumeau A, Fanello S. Acceptance of seasonal and pandemic a (H1N1) 2009 influenza vaccination by healthcare workers in a French Teaching Hospital. Vaccine 2011; 29(25):4190-4194.

31. Akker IL, Delden JJM, Verheij TJM, Essen GA, Sande MAB, Hulscher ME, Hak E. Which determinants should be targeted to increase influenza vaccination uptake among health care workers in nursing homes? Vaccine 2009; 4724-4730.

32. Hopman CE, Riphagen-Dalhuisen J, Akker IL, Frijstein G, Geest-Blankert ADJ, Danhof-Pont MB, Jager HJ, Bos AA, Smeets E, Vries MJT, Gallee PMM, Lenderink AF, Hak E. Determination of factors required to increase uptake of influenza vaccination among hospital-based healthcare workers. J Hosp Infect 2011; 77(4)327-331.

33. Payá JS, Garcia IH, Sañudo JB, Angeles RC, Rincon A, Candeira SR. Frecuencia de reacciones adversas y factores asociados tras la administración de la vacuna de la gripe en personal sanitario durante la temporada 2009-2010. Rev. Esp. Salud Pública 2010; 84(6):851859.

34. Bettinger JA, Rouleau I, Gariépy MC, Bowie WR, Valiquette L, Vanderkooi OG, Kellner JD, Coleman BL, McNeil SA, McCarthy A, Serres G. Successful methodology for large-scale surveillance of severe events following influenza vaccination in Canada, 2011 and 2012. Euro Surveill 2015; 20(29):1-9.

35. Center for Disease Control and Prevention (CDC). Seasonal Influenza Vaccine Safety: A Summary for Clinicians. Atlanta: CDC; 2015. [Internet].[acessado 01 nov. 2015]. Disponível em: http://www.cdc.gov/flu/ professionals/vaccination/vaccine_safety.htm 
36. Dedoukou X, Nikolopoulos G, Maragos A, Giannoulidou S, Maltezou HC. Attitudes towards vaccination against seasonal influenza of health-care workers in primary health-care settings in Greece. Vaccine 2010; 28(37):5931-5933.

37. Rachiotis G, Mouchtouri VA, Kremastinou J, Gourgoulianis K, Hadjichristodoulou C. Low acceptance of vaccination against the 2009 pandemic influenza a(H1N1) among healthcare workers in Greece. Euro Surveill 2010; 15(6):3.

38. Poland GA. The 2009-2010 influenza pandemic: effects on pandemic and seasonal vaccine uptake and lessons learned for seasonal vaccination campaigns. Vaccine 2010; 28(Supl. 4):3-13.

39. Rebmann T, Wright KS, Anthony J, Knaup RC, Peters EB. Seasonal influenza vaccine compliance among hospital-based and nonhospital-based healthcare workers. Infect Control Hosp Epidemiol 2012; 33(3):243-249.

Artigo apresentado em 21/05/2017

Aprovado em 09/02/2018

Versão final apresentada em 11/02/2018 